to be enlarged, a longer segment of the trachea would be necessary, and the primary section of the trachea would have to be much lower.

Care must be taken to connect the 2 tongues of the trachea, which must fit perfectly to the anterior part of the bronchi with no tension and no disrotation.

\section{Conclusion}

Extended slide tracheoplasties enable successful tracheal reconstruction even when main bronchi are involved in the hypoplasia. Use of the patient's own tracheal tissues and avoidance of foreign materials should enable good tracheobronchial growth in the same way that slide tracheoplasty enables good tracheal growth.

\section{References}

1. Tsang V, Murday A, Gilbe C, Goldstraw P. Slide tracheoplasty for congenital funnel shaped tracheal stenosis. Ann Thorac Surg. 1989;48:632-5.

2. Lang FJW, Hurni M, Monnier P. Long segment congenital tracheal stenosis: treatment by slide tracheoplasty. J Pediatr Surg. 1999;34:1216-22.

3. Kutlu CA, Goldstraw P. Slide tracheoplasty for congenital funnel shaped tracheal stenosis (a 9-year follow-up of the first case). Eur J Cardiothorac Surg. 1999;16:98-9.

4. Garabedian EN, Le Bret E, Corre A, Roger G, Pineau E, Bourel P. Tracheal resection associated with slide tracheoplasty for long segment congenital tracheal stenosis involving the carina. J Thorac Cardiovasc Surg. 2001;121:393-5.

5. Grillo HC (ed). Repair of congenital tracheal lesions. In: Grillo HC, ed. Surgery of the trachea and bronchi. Hamilton, Ontario, Canada: BC Decker Inc; 2004. p. 655-74

\title{
Methicillin-resistant Staphylococcus aureus infection causing Pancoast syndrome
}

Hon Chi Suen, MD, ${ }^{a}$ Richard A. Summa, MD, ${ }^{b}$ Alexander Babich, MD, ${ }^{c}$ Edwin C. Ernst, MD, ${ }^{d}$ Phillip Zinser, MD, ${ }^{e}$ Wilfrido C. Feliciano, MD, ${ }^{f}$ and John H. Rice, MD, ${ }^{g}$ St Louis, MO

I nfectious causes of Pancoast syndrome have been described. We present the first case report of methicillin-resistant Staphylococcus aureus (MRSA) pulmonary abscess with osteomyelitis of the first rib mimicking a Pancoast tumor in a non-immunocompromised young man.

\section{Clinical Summary}

A 41-year-old African American man reported right shoulder pain radiating down the right arm for 3 months. Examination revealed a well-built person with no positive physical findings. Chest radiography was unremarkable, but a computed tomography chest showed a 3-cm mass abutting the right first rib, right internal thoracic artery, and right brachiocephalic vein (Figure 1). There was no enlarged mediastinal lymph node. Positron emission tomography scan showed intense activity in the mass. Bone scan showed increased activity in the right first rib suggesting lytic changes. Magnetic resonance imaging of the chest confirmed chest wall invasion without vascular or brachial plexus involvement. The findings were indicative of a Pancoast tu-

From the a Departments of Cardiothoracic Surgery, ${ }^{\mathrm{b} P u l m o n o l o g y, ~}{ }^{\mathrm{c} P a t h o l-}$ ogy, ${ }^{\mathrm{d}}$ Radiology, ${ }^{\mathrm{e}}$ Infectious Disease, ${ }^{\mathrm{f}}$ Plastic Surgery, and ${ }^{\mathrm{g}}$ Internal Medicine, DePaul Health Center, St Louis, MO.

Received for publication Jan 18, 2006; accepted for publication Feb 8, 2006.

Address for reprints: Hon Chi Suen, MD, Cardiothoracic Surgery Associates, SC, 12B Park Place, Swansea, IL 62226 (E-mail address: HSUEN@ earthlink.net)

J Thorac Cardiovasc Surg 2006;132:183-4

$0022-5223 / \$ 32.00$

Copyright $\odot 2006$ by The American Association for Thoracic Surgery doi:10.1016/j.jtcvs.2006.02.023 mor. The initial plan was to confirm malignancy by needle biopsy and then treat the patient with neoadjuvant chemoirradiation followed by surgery. However, two needle biopsies were nondiagnostic.

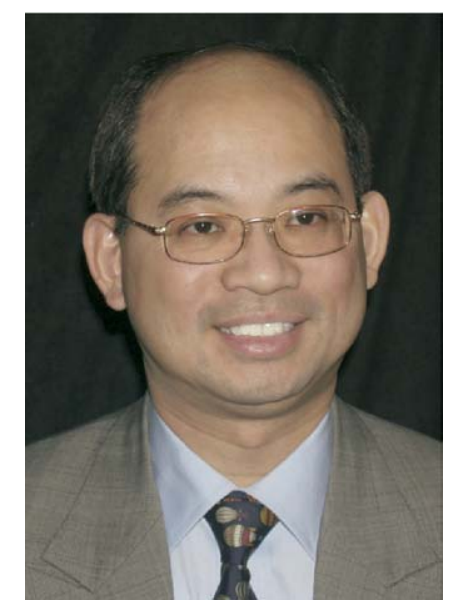

Dr Suen

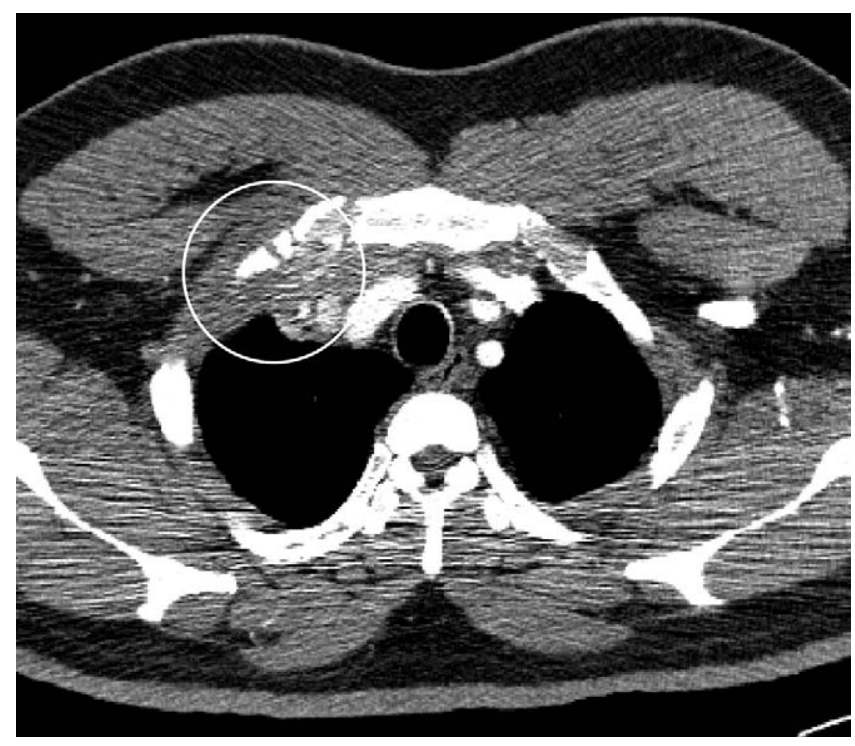

Figure 1. Computed tomography of the chest showed a 3-cm mass abutting the right first rib, right internal thoracic artery, and right brachiocephalic vein. 


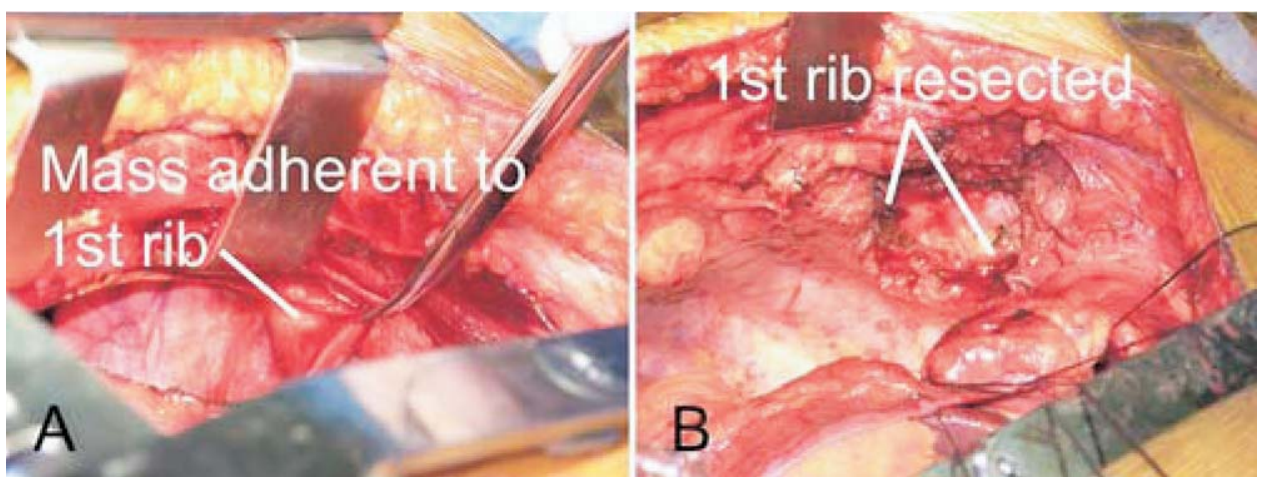

Figure 2. A, Right apical mass was firmly adherent to the first rib. B, Right first rib and neighboring part of manubrium were resected through median sternotomy.

After a negative bronchoscopy, the patient was explored through a median sternotomy. A Tru-cut biopsy of the lung mass and a biopsy of the right lower paratracheal and tracheobronchial angle lymph nodes were reported on frozen section to show non-small-cell carcinoma. We proceeded to perform an en bloc right upper lobectomy with first rib resection and mediastinal lymph node dissection. The mass was dissected from the brachiocephalic vein. The internal thoracic artery was resected with the mass. With an oscillating saw, the lateral part of the manubrium was resected within the chest cavity through the median sternotomy. The superior border of the first rib was found, and the periosteum was cauterized. A periosteal elevator was used to depress the first rib from the superior periosteum. The right subclavian vein and artery were protected by the superior periosteum of the first rib. The posterior aspect of the first rib away from the mass was transected with a rib cutter. The first intercostal muscles were transected on top of the second rib. Operative photographs before and after resection are shown in Figure 2. The right upper lobectomy and mediastinal lymph node dissection were completed.

The specimen was incised with a sterile blade revealing a small abscess in the middle of a 3- to 4-cm indurated mass. A culture of the pus grew MRSA. Permanent section of the mass and lymph nodes showed intense inflammation with no evidence of malignancy. The rich lymphoplasmacytic changes in the specimen were responsible for the misdiagnosis of malignancy on the frozen sections. The pulmonary infection resulted in osteomyelitis of the first rib. The patient was treated with long-term intravenous vancomycin.

The patient had been healthy. He was not a diabetic and did not use drugs. He was a nonsmoker and nondrinker, and had not been using antibiotics for at least 1 year before surgery. He worked in a prison and was human immunodeficiency virus negative.

His postoperative complications consisted of superficial sternotomy wound infection treated conservatively and right upper extremity venous thrombosis from an indwelling central line.

\section{Comment}

Various infectious agents have been reported to cause Pancoast syndrome. These include oxacillin-sensitive Staphylococcus aureus, ${ }^{1}$ Pseudomonas aeruginosa,${ }^{2}$ Pasteurella multocida, ${ }^{3}$ actino- mycosis, aspergillosis, nocardiosis, ${ }^{4}$ cryptococcosis, tuberculosis, mucormycosis, and hydatidosis. MRSA has never been reported to be a cause of Pancoast syndrome. We are still puzzled as to the cause of the MRSA lung abscess with osteomyelitis in a healthy, non-immunocompromised young man with no history of antibiotic intake or hospitalization.

This report again illustrates the importance of definitive pathologic diagnosis in patients who present with Pancoast syndrome. If a needle biopsy is negative for malignancy, a second biopsy should also be sent for various smears and cultures to rule out the many potential infectious causes of Pancoast syndrome. Intense antibiotic therapy has been reported to result in resolution of infectious Pancoast syndrome. ${ }^{3}$ In this man's case, even the frozen sections were misinterpreted as malignancy because of the severe inflammatory reaction.

Various surgical approaches have been described for resection of a Pancoast tumor. These include a posterolateral thoracotomy, an anterior cervicothoracic approach, ${ }^{5}$ and a trap-door approach (cervical incision, median sternotomy, and thoracotomy). This case demonstrates that en bloc right upper lobectomy and first rib resection can be accomplished through a standard median sternotomy alone. This represents another possible alternative approach to resection of an anteriorly located Pancoast tumor.

\section{References}

1. Gallagher KJ, Jeffrey RR, Kerr KM, Steven MM. Pancoast syndrome: an unusual complication of pulmonary infection by Staphylococcus aureus. Ann Thorac Surg. 1992;53:903-4.

2. Vandenplas O, Mercenier C, Trigaux JP, Delaunois L. Pancoast's syndrome due to Pseudomonas aeruginosa infection of the lung apex. Thorax. 1991;46:683-4.

3. Ribas J, Lores L, Ruiz J, Ausina V, Morera J. Pancoast's syndrome due to chronic pneumonia by Pasteurella multocida. Eur Respir J. 1997;10: 2904-6.

4. Larobina M, McLean C, Davis BB. Clinical-pathologic conference in general thoracic surgery: disseminated nocardiosis presenting as Pancoast syndrome. J Thorac Cardiovasc Surg. 2004;127:568-71.

5. Dartevelle PG, Chapelier AR, Macchiarini P, Lenot B, Cerrina J, Ladurie FL, et al. Anterior transcervical-thoracic approach for radical resection of lung tumors invading the thoracic inlet. J Thorac Cardiovasc Surg. 1993;105:1025-34. 